Anaesthesist 2013 · 62:169-170

DOI 10.1007/s00101-013-2149-y

Online publiziert: 10. März 2013

(c) Springer-Verlag Berlin Heidelberg 2013

\section{P. Kessler}

Abteilung für Anästhesiologie, Intensiv- und Schmerzmedizin, Orthopädische Universitätsklinik Friedrichsheim gGmbH, Frankfurt a. M.

\section{Intraneurale Injektion von Lokalanästhetika}

\section{Bedeutung für die erfolgreiche Nervenblockade}

Mit der klinischen Einführung der elektrischen Nervenstimulation in den 1970er Jahren wurde ein entscheidender Fortschritt in der peripheren Regionalanästhesie erzielt. Unabdingbare Voraussetzung für die bis dahin führende Parästhesietechnik war der direkte Nadel-NervKontakt. Beim blinden Vorschieben der Punktionskanüle war das Auslösen einer Parästhesie eine Conditio sine qua non für eine erfolgreiche Blockade.

Zunehmende Berichte über bleibende Nervenschäden nach dem Auslösen von Parästhesien sowie die Schmerzhaftigkeit des Verfahrens führten schließlich zur raschen Verbreitung der elektrischen Nervenstimulation und ließen sie zum Goldstandard der Punktionstechniken in der peripheren Regionalanästhesie avancieren. Mit dieser Technik glaubte man, das Aufsuchen peripherer Nerven reproduzierbar und absolut sicher durchführen zu können, ohne dass es zu einem NadelNerv-Kontakt mit eventuellem Kanülentrauma kommen muss.

Das zugrunde liegende Wirkprinzip der Nervenstimulation ist, dass ein von der Kanülenspitze abgegebener elektrischer Impuls über eine Nervenerregung eine motorische Reizantwort auslöst [4]. In Abhängigkeit von Stromstärke und Impulsdauer lässt sich so die Kanüle ohne Nadel-Nerv-Kontakt nah am Nerv platzieren. Aufgrund der positiven Korrelation von Nadel-Nerv-Abstand und Erfolgsrate wird eine möglichst nahe Platzierung der Kanüle und damit des
Lokalanästhetikums am Nerv angestrebt [7].

Die so millionenfach durchgeführten nervenstimulatorgestützten Nervenblockaden zeigen in Abhängigkeit von der Erfahrung des Anästhesisten und der Nervenlokalisation eine hohe Erfolgsrate zwischen 60 und fast $100 \%$ mit einer sehr geringen Inzidenz von bleibenden Nervenschäden (ca. 3-4/10.000 Blockaden, [3]). Hierbei wurden die seltenen Fälle neurologischer Defizite u. a. auf zu forsches Vorführen der Kanüle zurückgeführt.

Erste Zweifel an der Unfehlbarkeit der elektrischen Nervenstimulation kamen auf, als gezeigt werden konnte, dass das Auslösen von Parästhesien nicht zwangsläufig zu einer motorischen Reizantwort führt [9]. Völlig ins Wanken geriet die heile Welt der Nervenstimulation mit der Einführung der Neurosonographie Anfang der 1990er Jahre [5]. So löste ein im Ultraschall nachweisbarer Nadel-NervKontakt während einer axillären Plexusblockade nur in $38,2 \%$ eine Parästhesie und in nur $74,5 \%$ eine Muskelkontraktion aus [6].

Die neue Ultraschalltechnik brachte ein weiteres Phänomen zutage. Über die Visualisierung der Zielstrukturen und der Nadelspitze lässt sich die Ausbreitung des Lokalanästhetikums exakt verfolgen. So sah man anhand der Zunahme des Nervenquerschnitts, dass es gelegentlich $\mathrm{zu}$ einer intraneuralen Ausbreitung des Lokalanästhetikums kommt, ohne dass $\mathrm{Pa}$ - tienten Parästhesien oder Schmerzen bei der Injektion angeben oder über postoperative neurologische Defizite klagen.

Basierend auf dieser Beobachtung publizierte Bigeleisen [2] 2006 eine Studie, bei der im Rahmen einer axillären Blockade Lokalanästhetika bewusst intraneural injiziert wurden. Trotz nachweislicher intraneuraler Injektion wies keiner der Patienten ein postoperatives neurologisches Defizit auf.

Im Kontext mit der seither heftig und kontrovers geführten Diskussion über den Stellenwert einer intraneuralen Injektion von Lokalanästhetika steht die in dieser Ausgabe von Der Anaesthesist publizierte Arbeit von Seidel et al. Die Autoren zeigen u. a., dass die ultraschallgesteuerte intraneural-perifaszikuläre Ausbreitung des Lokalanästhetikums bei der distalen N.-ischiadicus-Blockade eine hohe Erfolgsrate aufweist, ohne dass postoperativ Nervenschäden nachweisbar sind.

Ist diese Vorgehensweise nun ein sicherer zukunftweisender Weg einer erfolgreichen Regionalanästhesie?

Keinesfalls. Die Datenlage ist zu dünn, um diese Frage wissenschaftlich fundiert bejahen zu können. Schließlich steht mit der herkömmlichen extraneuralen Lokalanästhetikaapplikation eine sichere Alternative, zwar mit etwas verlängerter Anschlagzeit, zur Verfügung. Weiterhin ist unter dem Aspekt der Sicherheit eine Grundvoraussetzung bei der intraneuralen Injektion von Lokalanästhetika nicht erfüllt. So liegt das Auflösungsvermögen 
der Ultraschallwellen in Abhängigkeit von der Schallfrequenz bei etwa $1 \mathrm{~mm}$. Damit ist eine sichere extrafaszikuläre Nadelposition mit dem Ultraschall nicht detektierbar, da Faszikel oft einen geringeren Durchmesser aufweisen [8]. Somit bleibt eine intraneural-intrafaszikuläre Kanülenposition möglich und damit das Risiko eines Nervenschadens bestehen. Hinzu kommt, dass die Darstellbarkeit intraneuraler Strukturen im Ultraschall derzeit nur bei oberflächlichen Nerven gelingt.

Hinsichtlich des Auslösens von Parästhesien bestätigt die Untersuchung von Seidel et al. (s. S. 183), dass diese häufiger bei ultraschallgesteuerten als bei nervenstimulatorgestützten Blockaden auftreten und teilweise von den Patienten als äußerst unangenehm sowie schmerzhaft empfunden werden. Entscheidend trägt dazu die fehlende Visualisierung der Kanülenspitze beim Vorschieben der Nadel bei; dies ist oftmals Folge eines ungenügenden Trainings- oder Ausbildungszustands.

Ein weiterer Verdienst der Autoren ist es, mit der vorgestellten Arbeit die aktuelle Diskussion über die Nomenklatur einer extra- und intraneuralen Injektion von Lokalanästhetika aufzugreifen. So hat die Neurosonographie in den vergangenen Jahren unser anatomisches Verständnis über die Mikroarchitektur peripherer Nerven deutlich erweitert. Binnen- und bindegewebige Hüllstrukturen der Nerven lassen sich jetzt besser zuordnen oder werden, wie am Beispiel des N. ischiadicus, mit „Paraneurium“ neu definiert [1]. Für Klarheit sorgt hier die vorgestellte Arbeit dadurch, dass die intraparaneurale Injektion des Lokalanästhetikums in den $\mathrm{N}$. ischiadicus definitionsgemäß als extraneural in Bezug auf die Nn. tibialis und fibularis - beides eigenständige Nerven bezeichnet wird.

Ebenso werden die bereits erwähnten Limitationen der elektrischen Nervenstimulation aufgezeigt. Die vorliegenden Ergebnisse bestätigen, dass es trotz des Einsatzes der Nervenstimulation, häufiger als früher vermutet, zu einer ultraschallgesicherten intraneuralen Kanülenposition kommt, ohne dass dies von den Patienten als Parästhesie oder als Schmerz bei der Injektion registriert wird oder mit einem postoperativen neurolo- gischen Defizit korreliert. Möglicherweise disloziert die Kanülenspitze unter der Injektion nach extraneural, sodass lediglich geringe Mengen von Lokalanästhetika intraneural und somit folgenlos für den Patienten appliziert werden. Andererseits lag die benutzte Stromstärke mit 0,2 mA unter der allgemein empfohlenen Schwellenstromstärke von 0,3-0,5 mA, was eine Erklärung für die hohe Anzahl an Parästhesien wäre.

Zweifelsohne stellen die Ultraschallverfahren eine echte Bereicherung im regionalanästhesiologischen Alltag dar und haben unser Verständnis über die elektrische Nervenstimulation erweitert. Damit die Erfolgsstory des Ultraschalls und der Regionalanästhesie weiterhin bestehen bleibt, sollten die bis jetzt vorliegenden Daten zur intraneuralen Injektion von Lokalanästhetika als Ziel einer erfolgreichen Nervenblockade vorsichtig und zurückhaltend interpretiert werden. Was für den distalen N. ischiadicus mit seinem Paraneurium zutrifft, ist auf andere nervale Strukturen oder Regionen nicht ohne Weiteres übertragbar. Ungeklärt ist auch folgende Frage: Welches intraneurale Volumen oder welche intraneurale Konzentration an Lokalanästhetikum verträgt ein Nerv? Von daher kann derzeit die routinemäßige intraneurale Injektion von Lokalanästhetika als Verfahren zur Nervenblockade nicht empfohlen werden. Sobald im Ultraschallbild eine intraneurale Nadellage oder ein Anschwellen des Nervs unter der Injektion beobachtet wird, sollte die Injektion gestoppt und die Punktionskanüle zurückgezogen werden.

Mit Spannung darf man den weiteren experimentellen und klinischen Daten zu dieser Thematik entgegensehen.

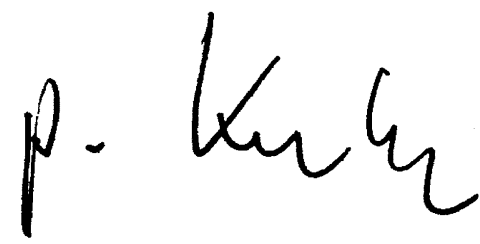

P. Kessler

\section{Korrespondenzadresse}

Prof. Dr. P. Kessler

Abteilung für Anästhesiologie, Intensiv- und Schmerzmedizin, Orthopädische Universitätsklinik Friedrichsheim gGmbH Marienburgstr. 2, 60528 Frankfurt a. M. p.kessler@friedrichsheim.de

Interessenkonflikt. Der korrespondierende Autor weist auf folgende Beziehungen hin: Vortragshonorare von B. Braun, Pfizer + Bayer.

\section{Literatur}

1. Andersen $\mathrm{HL}$, Andersen SL, Tranum-Jensen J (2012) Injection inside the paraneural sheath of the sciatic nerve. Reg Anaesth Pain Med 37:410 414

2. Bigeleisen PE (2006) Nerve puncture and apparent intraneural injection during ultrasound-guided axillary block does not invariably result in neurologic injury. Anesthesiology 105:779-783

3. Brull R, McCartney CJ, Chan VW, El-Beheiry H (2007) Neurological complications after regional anesthesia: contemporary estimates of risk. Anesth Analg 104:965-974

4. Kaiser H, Niesel HC, Hans V (1990) Grundlagen und Anforderungen der peripheren elektrischen Nervenstimulation. Ein Beitrag zur Erhöhung des Sicherheitsstandards in der Regionalanästhesie. Reg Anesth Pain Med 13:143-147

5. Kapral S, Krafft P, Eibenberger K et al (1994) Ultrasound-guided supraclavicular approach for regional anesthesia of the brachial plexus. Anesth Analg 78:507-513

6. Perlas A, Niazi A, Mccartney C et al (2006) The sensitivity of motor response to nerve stimulation and paresthesia for nerve localization as evaluated by ultrasound. Reg Anesth Pain Med 31:445-450

7. Raj PP, Rosenblatt R, Montgomery SJ (1980) Use of the nerve stimulator for peripheral blocks. Reg Anaesth 5:14-21

8. Silvestri E, Martinoli C, Derchi LE et al (1995) Echotexture of peripheral nerves: correlation between US and histologic findings and criteria to differentiate tendons. Radiology 197:291-296

9. Urmey WF, Stanton J (2002) Inability to consistently elicit a motor response following sensory paresthesia during interscalene block administration. Anesthesiology 96:552-554 